\title{
Design and implementation of USB_RS232 interface converter
}

\author{
Haoran Song \\ Marine college of Shandong jiaotong university, Weihai, Shandong,China
}

Keywords: Universal serial bus, RS232 interface, Single-chip microcomputer, the driver, the converter

\begin{abstract}
With computer of development, especially hardware technology of development, traditional of RS232 serial interface has displayed out increasingly more of insufficient, but large of instrument instrument, and industrial control system, and remote data terminal, equipment still using RS232 serial interface and variants as main of communications way, this and current popular host of external equipment interface way not phase adapted, paper proposed a USB interface to RS232 interface seamless conversion of interface conversion device of design programme, the programme based on single tablets machine achieved, And can directly use its original traditional equipment RS232 interface for two-way data transfer with the USB host-bus interface. The scheme has double buffering function, Plug and play, easy to use, cost-effective high.
\end{abstract}

\section{Introduction}

Computer peripheral interfaces after years of development, there have been great changes. Traditional interface includes two serial ports and one parallel port, serial port RS232C Protocol, speed range is $0-20 \mathrm{kbps}$, and later extended to $115 \mathrm{kbps}$. Serial ports are used to connect the mouse, modem and other equipment. Serial and parallel ports, although slow speed, but because of their simple lines, transmission distance, along with the computer into the industrial control field, the serial interface is widely used. And mainly used to connect printer standard parallel 8-bit data width and speeds of $1 \mathrm{Mbps}$, much faster than a serial port. Standard and later extended to the EPP and ECP mode parallel port can be bi-directional data transfer. But because parallel lines complicated, data transmission, are subject to interference, it only used to connect host peripheral devices, such as printers, scanners, plotters, etc.

In recent years, the significant increase in the speed of your computer and peripherals of big increase in variety and quantity, peripherals peripheral interfaces of the host's speed, ease of use put forward higher requirements. As requirements grow, traditional serial and parallel ports can not meet the needs of development. Some hosts began to change the original design, one is adding new peripheral interfaces, such as USB, FireWire bus. The other hand reduces the number of traditional interfaces, and cancel, reduce serial port to 1 or cancel. But is widely used due to the RS232 serial interface, its type of bus RS485, RS422 and so on, have a lot of control equipment, instrumentation, industrial control systems, remote terminals with serial interfaces. The reduction or even elimination of serial interface, and extensive application of serial interface between a very big contradiction.

The wide application of USB interface, and cannot be immediately eliminated the serial bus interface. USB interface is completely different from the traditional interface on the one hand, it is a software-based bus, with complex data formats, and protocols. Thus, devices support USB interface, not only to connect hardware, software design, a great deal of work remains to be done. The other hand, RS232 serial interface very easy to use, interface device driver provided by the operating system, the program simply set bits, stop bits, data bits, test transmission rate, and then calls the appropriate system functions to communicate with the device. 


\section{USB universal serial bus protocol}

USB universal serial bus is a new kind of computer peripheral interface bus, which is designed for traditional computer external interface is not Plug-and-play functionality and does not support hot-plug, less slow, does not support the automatic configuration of the shortcomings, and design a has an autoconfiguration capability, expanded easily, comes with power, the transmission speed of the new interface. USB universal serial bus USB bus connections, connections between devices on the USB bus and USB hosts, is an integrated system. USB concept refers to the data bus connections on the device and the operation of transmission between hosts, this includes multiple content: the entire USB bus topology; the specific methods of data transmission between the device and the host; USB internal distribution and response rules transfer mode USB bus data stream; scheduling and allocation of all data transfer bandwidth USB bus.

A USB system, there can be only one host at any point. All operations are initiated by the host. But a computer can have more than one USB host, no restrictions on this. USB host computer is called the host controller, host controllers are not just hardware, this concept also includes software, it actually is a synthesis of hardware and driver software. Integrated root hub on the host, used to extend bus root hub can be directly connected to an external device, you can also connect to other hubs and hubs connect to other external devices. USB host structure as shown in Figure 1.

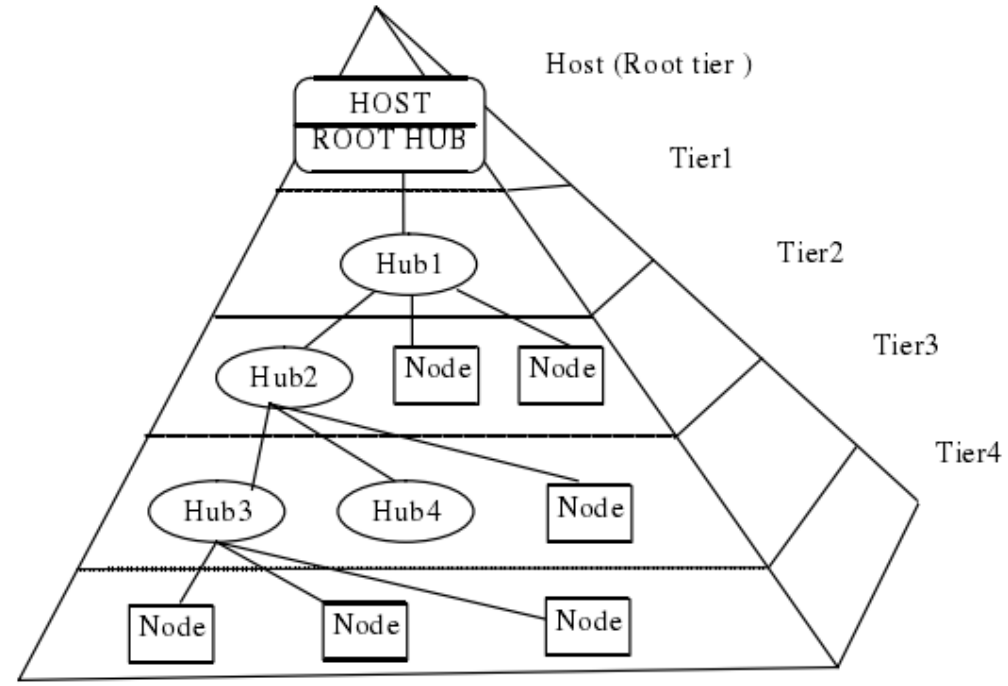

Fig . 1 The topology of the USB

USB host manages the USB channel, it requires the driver to complete channel management. There are two kinds of drivers in the system, USB host driver is responsible for complete control of the host controller, it also accepts requests from upper-layer USB drivers, and complete the appropriate action. Finally, under software control, completed by the host controller interacts with the physical bus data.

USB bus protocol describes how to complete the data transfer between host and device, and provided for all transfer details, these details include how interactions between host and device interaction how to complete, what kind of syntax and Protocol. Also, USB bus protocol also establishes, fields, packages, services, structure of the transmission, and defines how fields constitute a package, package, what constitutes a transaction, the transaction constitutes a transfer.

\section{USB-RS232 interface converter circuit design}

The basic principle of USB-RS232 interface converter is that when the host sends, the converter receives data from host USB interface, and then sent from the RS232 interface to the device. At send time, converter receives the data from the RS232 interface, and then is sent to the host from a USB interface, this completes the conversion interface. In the course of data flowing through the converter, the converter to buffer data to coordinate the two interfaces differ in rate. 
According to the functional requirements of the USB-RS232 interface converter. Integrated within CPU used in the scheme, watchdog circuit, reset circuit, data storage, memory and other components of the microcontroller. Connect the USB to the USB bus interface using common interface chips. The programme has the advantage of simple structure, electrical connection, system integration, high, small, system debugging relatively easy.

SCM uses to access external memory access instructions D12. Other control signals related to a total of 5, were issued by the microcontroller, these signals are address latch, D12ALE, chip/D12CS, read operations/D12RD,/D12WR, reset the write/D12RST. Interface IC power provided by the USB interface $5 \mathrm{~V}$ power supply.

D12 signal D12INT is for single-chip interrupt request signal, the signal is issued when D12 when the data is received or sent to notify SCM, D12 has USB transactions took place. When an interrupt occurs, the single-chip calls an interrupt service routine, D12 was the appropriate action in the procedure, handle the USB transactions. Just insert the USB interface converter interface, device enumeration. Device enumeration, connecting pins LED light emitting diode will blink on the GOODLINK, which can be used to indicate the connections D12. Interface converter enumeration is successful, the LED light-emitting diodes will be permanent. If the interface converter is pending, this light will be off.

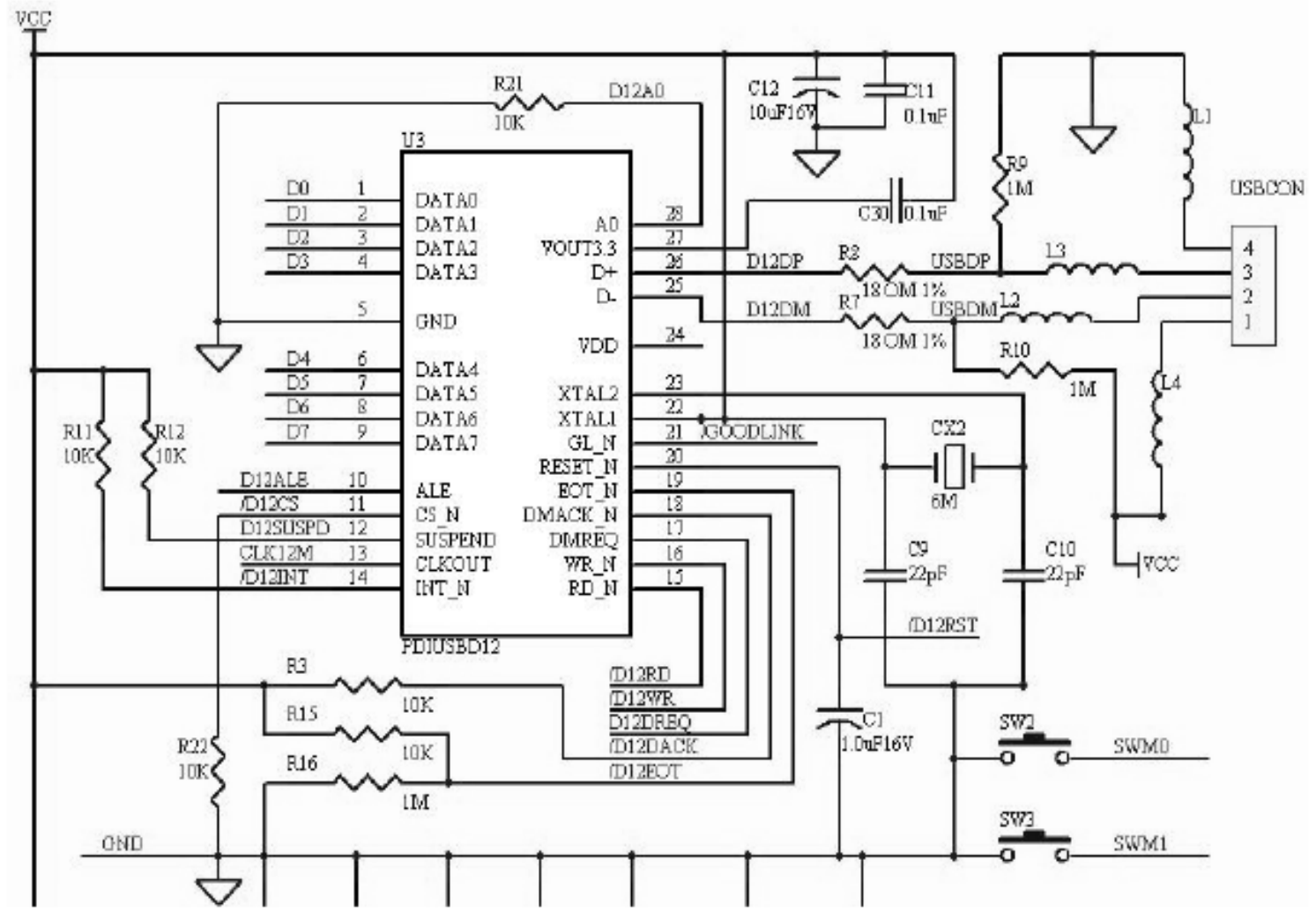

Fig . 2 USB bus interface circuit

\section{Design of USB device driver}

Firmware includes the main loop, USB interface chip interrupt service routine, standard USB device request processing, realization of serial interface interrupt processing and encryption algorithms. Initialization procedures complete MCU internal parts and the initialization of the USB interface chip. USB interrupt service routines processing interrupts from the USB interface chip, to deal primarily with USB. Main parts awaiting the arrival of interruption, according to interrupt flag to enter the corresponding subroutine, separate bus reset, suspend, change, control transmission operation and data transfer operations.

Microcontroller firmware's main function is to communicate with the USB interface chip, resolve USB protocol requests and works with host USB device drivers, complete with communications 
applications, complete enumeration and identification of the interface converters, data protocol conversion work is completed.

Modern operating systems limit behavior for the application, and to the protection of the operating system itself. In the Windows operating system, restricts access to applications, only the driver can access the interface converter directly. Therefore, the application converter to interface device device drivers, then access to the device by the driver. Converter USB device driver design based on WDM driver model.

USB devices and generic hardware device, USB device is more of a logical device, USB device drivers do not directly control your USB devices. USB device driver, use the following method to handle the user request. According to user requirements, establish an URB (USB request block), which contains information on user request. URB structure is then sent to the USB bus driver, USB bus driver is provided by the host, it parses the URB, operational requirements for completion of URB, and finalize the communication with a USB hardware device.

Device object of the stack building is done by the operating system plug and play Manager, the Manager sent to it according to the driver's request to decide how to set up device stack. General works as follows: after the new devices when the system starts or when the bus, the bus driver will connect his physical to enumerate devices on the bus. In the enumeration process, it finds a physical device, will be the establishment of a corresponding device objects, these devices are called physical device object for PDO.

The plug and play Manager loads to start driver. It to find the driver file, it loads the system nonpaged memory and interface converter driver's AddDevice function is executed. The main functions of this function is to create a device object that corresponds to the interface converters, connect to the device and to use the object on the stack. AddDevice function device interface, but also to create a symbolic link, interface converters to the application and exposes the driver interface of the system, the system can be based on this interface, the driver sends requests to the interface converter.

USB bus driver by creating a structure of URB, sends the host controller to complete the job. Host controller via the USB bus converter to issue configuration commands to the interface requires select configuration and interfaces interface converter, the request by the interface converter in the microcontroller program is responsible for processing. Application software in the Windows operating system to access the system through the handle object, application software to access the interface converter, you need to establish connection to interface converter pipe system, and get the handle to the pipe. There are two handles in the system, configure the handle for use by the application when the command is sent to the device configuration, interface handle application software and interface converter implementation used during data transfer.

\section{References}

[1]Hazzah Karen.Writing Windows VxDs and Device Drivers Second edition[M].R\&D Books,1997.87-93.

[2]Wang Chengru, Li Ying-wai.. Principle and engineering of USB2.0 development [m]. Beijing: national defense industry press, 2004.7:125-126

[3]John Shumate. Apraetical Guide to Microsoft 0LAP Server[M]. Addisonwesley. 2000

[4]Gu Yan. based on data warehouse technology in hospital information systems (HIS) Scheme [j]. computer systems applications. 2005

[5]Yi Jing. and implementation of data mining technology in the hospital information [j]. Chongqing University of medical sciences 2007.

[6]Kantardzic M. Data Mining Concept、Models、Methods and Algorithms[M]. IEEE Press.2002 
[7] Bryan Ford, Pyda Srisuresh, and Dan Kegel. Peer-to-Peer Communication Across Network Address Translators. In USENIX Annual Technical Conference, Anaheim, CA, April, 2005.

[8] Rosenberg J. Schulzrinne H.Camarillo G.Johnston.A.Peterson , J.Sparks , and E.Schooler. SIP:Session Initiation Protocol. RFC 3261， June 2002.

[9] Hill R,Wang J,Nahrstedt K.Quantifying Non-functional Requirements:A Process Oriented Approach[C]. IEEE International Requirements Engineering Conference, Kyoto, Japan, 2004: 352-353 